\title{
Flip \& Pair - a strategy to augment a blended course with active-learning components: effects on engagement and learning
}

\author{
Vijayanandhini Kannan ${ }^{1 *}$, Hiroyuki Kuromiya ${ }^{2}$, Sai Preeti Gouripeddi ${ }^{1}$, Rwitajit Majumdar ${ }^{3}$,
} Jayakrishnan Madathil Warriem ${ }^{4}$ and Hiroaki Ogata ${ }^{3}$

\author{
*Correspondence: kvnandhini@ \\ gmail.com; vkannan@gitam.edu \\ 'Department of Physics, GITAM \\ School of Sciences, GITAM (Deemed \\ to be University), Rudraram, \\ Hyderabad 502 325, India \\ Full list of author information is \\ available at the end of the article
}

\begin{abstract}
Blended learning technique has adapted many new digitized tools to facilitate students with flexible and self-phased learning opportunities. The flipped classroom strategy, one of the blended learning models has been limited by low engagement of students in the online component. In the present study, we augment a Flip and Pair (F\&P), an active-learning strategy into the blended learning course. Following the $A B$ type single group quasi-experimental design, we evaluated the effects of F\&P strategy on the student's engagement and learning while orchestrating it for an undergraduate engineering physics course. Our results highlighted that there is a positive correlation between the engagement (computed based on learning logs of TEEL (Technology-enhanced and Evidence-based Education and Learning) platform in the F\&P activities with that of the performance score (knowledge quizzes and final exam). F\&P strategy had a better contribution compared to Flip and Traditional Teaching (F\&T) strategy with respect to both engagement and performance. Also, students exhibited a positive perception of learning and engagement. Based on our findings, we identified the key instructional measures that an instructor can follow to increase student engagement while using the F\&P strategy.
\end{abstract}

Keywords: Flip \& Pair strategy, Blended learning, Learner-centric MOOCs (LCM), Technology-enhanced evidence-based education and learning (TEEL), Student engagement

\section{Introduction}

Blended learning is an umbrella term used to define the broad teaching approaches that integrate the effectiveness of both the face-to-face (f2f) classroom and the technology-enhanced learning environments (Hrastinski, 2019; Yılmaz \& Malone, 2020). Blended learning utilizes a wide range of information and communication technology tools such as the learning management system (LMS), knowledge building applications like blogs, Wikis, forum, concept mapping tools, online authoring tools, open educational resources (OERs) (Garrison \& Vaughan, 2008), etc. Integration of

(c) The Author(s). 2020 Open Access This article is licensed under a Creative Commons Attribution 4.0 International License, which permits use, sharing, adaptation, distribution and reproduction in any medium or format, as long as you give appropriate credit to the original author(s) and the source, provide a link to the Creative Commons licence, and indicate if changes were made. The images or other third party material in this article are included in the article's Creative Commons licence, unless indicated otherwise in a credit line to the material. If material is not included in the article's Creative Commons licence and your intended use is not permitted by statutory regulation or exceeds the permitted use, you will need to obtain permission directly from the copyright holder. To view a copy of this licence, visit http://creativecommons.org/licenses/by/4.0/. 
technologies in blended learning provides timely and constructive feedback, thereby, transforming the instructor-centric education into a student-centric one (Bernard, Borokhovski, Schmid, Tamim, \& Abrami, 2014). With the increasing affordability of electronic gadgets and familiarity in different digital learning environments, students are now able to realize the benefits of Blended Learning (Buket \& Meryem, 2008; Glazer, 2011).

Though blended learning promotes a self-paced and flexible learning environment, it mostly depends on the individual's self-motivation to take responsibility for their own learning (Chew \& Wee, 2015). Research on flipped classroom strategy, a model of implementing blended learning, has provided pieces of evidence that students often exhibit poor engagement, lack of self-regulation and time management skills, and thereby limit the possibility of reaping the benefits of the learning strategies (Rasheed et al. 2020; Halverson, 2016). This is particularly important while adopting similar strategies in foundational courses like Physics. Physics is generally perceived as one of the most problematic subjects among all other science disciplines for high school students and becomes even more evasive when they enter into tertiary level education (Erhan, 2016). Though blended learning strategy has proven to be effective to support the student learning, augmenting a flipped classroom within the f2f physics courses has remained a challenge (Hamdan, McKnight, McKnight, \& Arfstrom, 2013). The context of this study is a Physics undergraduate course where we explore the use of flipped learning approaches and provide the experience of blended learning to the students.

Researchers have reported that use of innovative online tools combined with appropriate focus on student interactions in a blended physics course increased the students learning capacity and boosted a high-level of self-reported enjoyment (Ramma, Bholoa, Watts, \& Nadal, 2018). There are also studies that point to the critical nature of student's online engagement for achieving the course outcomes like critical thinking abilities and practical competence (Trowler \& Trowler, 2010). Careful design of online activities to engage students in a flipped setting allows teachers to plan the f2f sessions more effectively to engage students at higher-order learning activities (Beer, Clark, \& Jones, 2010). However, most teachers face both intrinsic and extrinsic barriers - access to relevant technology tools and support, attitude and belief towards technology, ability to design lessons that promote learner engagement and critical thinking that limit the adaptation of new technological tools for an effective blended learning experience (Johnson, Jacovina, Russell, \& Soto, 2016). While the extrinsic problems can be tackled through policy and institutional support, the intrinsic problems require multi-pronged strategy.

In the current study, we explore the design of a flipped learning experience in an undergraduate physics course by focusing on promoting learner engagement in both online (flipped) and classroom (face-to-face) settings. This design is informed by existing researchbased and is supported by a technology environment that assists the teacher with critical analytics information. The larger research objective of this paper is to look at how learning designs for engagement increase the effectiveness in a blended learning setting. The paper is organized as follows: The section II, describes the existing literature, Section III analyzes the pedagogical and technological foundation of current work and the Flip \& Pair (F\&P) orchestration strategy, section IV explains the research methods and data collections used to evaluate the effectiveness of the F\&P strategy. Section V \& VI presents the results and discussions respectively. Along with the results, we provide some reflections from the instructor perspective while adapting the F\&P strategy, limitations and future work. 


\section{Related literature}

The flipped classroom is one of the popular blended learning models adopted by educators worldwide (Hamdan et al., 2013). Application of flipped model in the physics domain has shown that it works well for the motivated students to increase their ability to learn in a self-paced manner. Researched pedagogical strategies designed to inculcate reflective learning practice is one of the possible solutions to achieve the learning goals and outcomes of flipped classroom strategy (Hamdan et al., 2013). Effective Integration of digitalized tools within the flipped learning can be further supported to strengthen and create more flexible teaching-learning environment. Here, we first highlight different technology based AL interventions that are reported in the literature in context of flipped online learning. Next, we provide references to various AL strategies designed for the f2f classroom, particularly, in the context of physics education.

\section{Technology tools for flipped classroom}

The flipped model utilizes a variety of technology tools to engage learners outside the classroom and then require teachers to engage students in higher order activities inside the classroom (Henrie, Halverson, \& Graham, 2015). Videos are one popular technology tool used in flipped learning. Studies have highlighted that educational videos could effectively enhance conceptual attainment and assist students to illustrate hard-tovisualize phenomena (Guo, Kim, \& Robin, 2014). To enhance learning from an educational video, it is suggested to keep videos targeted on learning goals (Hsin \& Cigas, 2013), reduce cognitive load by designing a short lecture video and avoid abrupt transitions (Kim et al., 2014). Few other studies have reported that embedding interactive videos with guiding questions maximizes the student retention and also act as an implicit way of sharing the learning outcomes (Lin et al, 2017; Brame, 2015). There are also other technology tools like simulations and e-text provided through learning management systems that have been attempted by instructors (Chen, Stelzer, \& Gladding, 2010; Sadaghiani, 2011). Use of web-based instructional tools has invigorated interest in data-driven education due to the scope to collect and analyze large data using technology platforms (Imran, 2019; Kay, 2011). Rachel et al. (2020) have provided insights on how to utilize the click-stream data tool to appropriately shape the instructional design of a lecture video and to examine the student's self-regulated learning. Recently Ogata et al., (2018) have developed the learning analytics tools to extract pieces of evidence from log data and to harness the power of technology-enhanced teachinglearning practice considering contextual teaching-learning practices.

In an earlier research, Sadaghiani (2011) had provided empirical findings on how the web-based content such as the multimedia learning modules, computer simulations, and e-Text improved student engagement in a physics course. The blended course had students attempt the e-content and followed it up with a mini-assignment (in the form of questions) before moving to f2f classroom sessions (Linghong \& Tang, 2017; Teheran et al., 2010). Yet another study in the physics domain shows that the web-based pre-lectures developed using the multimedia learning modules prepare the student better for a $\mathrm{f} 2 \mathrm{f}$ introductory physics lectures and also makes the course less difficult (Chen et al., 2010; Stelzer et al., 2010). This study also utilized a similar methodology of following up 
e-content with mini assignment questions (termed pre-flight questions) to ensure conceptual clarity. These studies show that using the digitalized tools alone does not automatically guarantee an improved learning effectiveness or student satisfaction and there has to be appropriate pedagogical designs that allow teachers to dynamically adapt the instructional strategy (Ferreira, de Oliveira, \& Araújo, 2019).

\section{Active learning in face-to-face class of flipped classroom}

The research conducted especially for the tertiary level undergraduate physics courses has shown that it is quite difficult to learn physics with the traditional teaching (TT) method (Bach, 2007; Bonwell, 1996). One of the major challenges being faced is to engage students in the higher order tasks using the TT method. With the monotonous mode of f2f lecturing and a passive listening tendency, many students get distracted easily to off-task activities. In contrast, the AL strategies implemented within the f2f class demands the students to get into a sustained mode of engagement and learning (Mintzes \& Walter, 2020). Thus, the researchers have employed various AL pedagogies to facilitate the students to participate actively in the $\mathrm{f} 2 \mathrm{f}$ class and to remove any learning difficulties by way of collaborative and reflective learning practices. With these interactive learning strategies, there are reports that highlights on a positive shift in the student's attitude and beliefs about learning physics subject (Kannan \& Gouripeddi, 2018; Zhang, Ding, \& Mazur, 2017). Some of the innovative f2f instructional strategies includes the peer instruction (PI) (Crouch, \& Mazur, 2001), project-based learning (Barron et al., 1998), think-pair-share (Raba, 2017; McTighe \& Lyman, 1988), adaptive learning (Peter Brusilovsky, 2003), jigsaw method (Aronson \& Patnoe, 1997), etc., all of which are based on cooperative learning.

Several educational researchers have substantially proven with evidence that the PI pedagogy offer a great potential to significantly improve the learning as compared to the TT, in context to the Physics courses at college or University (Meltzer, \& Thornton, 2012). Empirical studies of PI methods have shown that it helps to solve any specific learning difficulties in the Physics concept and the students taught via PI score significantly higher grades than an equivalent traditional teaching method (Meltzer, \& Thornton, 2012). Another instance on the research conducted with the Jigsaw method, one of the cooperative learning models has shown a better academic achievement in case of the undergraduate Physics laboratory practices as compared to confirmatory laboratory method. Tanel \& Enrol (2008) have analyzed the experimental teaching sequence using the jigsaw technique to Physics courses and established that the students who have difficulty to learn difficult concepts such as magnetism or electricity were able to learn better. More recent study on the impact of Team-based learning (TBL) in an introductory Physics course, indicate that TBL is a less intimidating classroom format (Michele, Rose, George, \& Zuleyha, 2020). Their findings also showed that the students appreciate the instant feedback as it helped them to learn more quickly and kept them engaged in $\mathrm{f} 2 \mathrm{f}$ class. They expressed that the peer explanations in the TBL method to be the most valuable to keep them motivated and accountable.

To summarize, we see that some format of peer learning is seen as beneficial strategy for Physics classrooms for entire set of students. Thus if we were to couple the technology based out-of-class sessions with peer-learning based f2f sessions, there is a high likelihood of meaningful learning happening for our learners. 


\section{Motivation}

The context being described in the current paper is to implement flipped learning for a Physics course of regular undergraduate engineering program in Indian University. The context is particularly challenging due to the instructor-mediated nature of regular f2f sessions, strict technology policies implemented within the institution and the attitude of learners towards Physics course. The current academic policies related to use of technology within the classroom restrict teachers to make use of only presentation technologies (Computer and Projector). Effective blending of research-based pedagogies with technology-mediated learning and then to follow-up with a corrective measure becomes extremely laborious. Further, even though knowledge of fundamental Physics principles is core to the understanding of engineering applications, Engineering Physics is often treated by students as a mandatory first year subject that they have to successfully pass. These facts make the adoption of AL strategies within a flipped classroom setting in the present context meaningful.

To successfully blend technology and f2f sessions we adopt a Flip \& Pair (F\&P) pedagogy within our flipped classroom course. In case of F\&P, in addition to the flip phase online learning, there were several regular $f 2 f$ classes. Both the f2f class and the flip online phase had an active-learning component as a possible solution. For example, the material in flip phase was provided with the learning activities based on learner-centric MOOCs, (LCM) pedagogical model and during the f2f class, we adapt the PI strategy. The authors have previously reported the preliminary observations on adaption of LCM pedagogy in context to blended Physics course (Kannan \& Gouripeddi, 2019). Further, a case study of the data-driven validation of the blended Physics course were reported using the learning logs of students (Kuromiya, Majumdar, Warriem, \& Ogata, 2019). The present work mainly focuses on orchestrating the LCM pedagogy with the support of learning analytics (LAView) dashboard tool of TEEL (Technology-enhanced and Evidence-based Education and Learning) platform. The TEEL platform provide us with several technological affordances in terms of creating evidences for student engagement while the LCM pedagogy assists to curate the contents within the AL environment. Our present study would address to some of these challenges and tries to bring-in a better relevance to the domain of physics education in context with the tertiary level engineering program in India.

\section{Description of the Flip \& Pair (F\&P) orchestration strategy}

\section{Creating blended contents using learner-centric MOOCs}

In the current work, we adopt the Learner-Centric MOOCs (LCM) model as an instructional design guide to design the flipped contents in the F\&P strategy and utilize the learner analytics dashboard tools to analyze its effectiveness. The LCM model is "a prescriptive model consisting of a set of guidelines, activity formats and actions for MOOC creators" proposed by Murthy et al. (2018). The model emphasizes interactive activities rather than traditional information transfer and maintains a learner-centric pedagogy as its main orchestration. Figure 1 depicts the overview of the pedagogical basis of the LCM model that consists of four activelearning structural components with a unique interaction line as follows. 


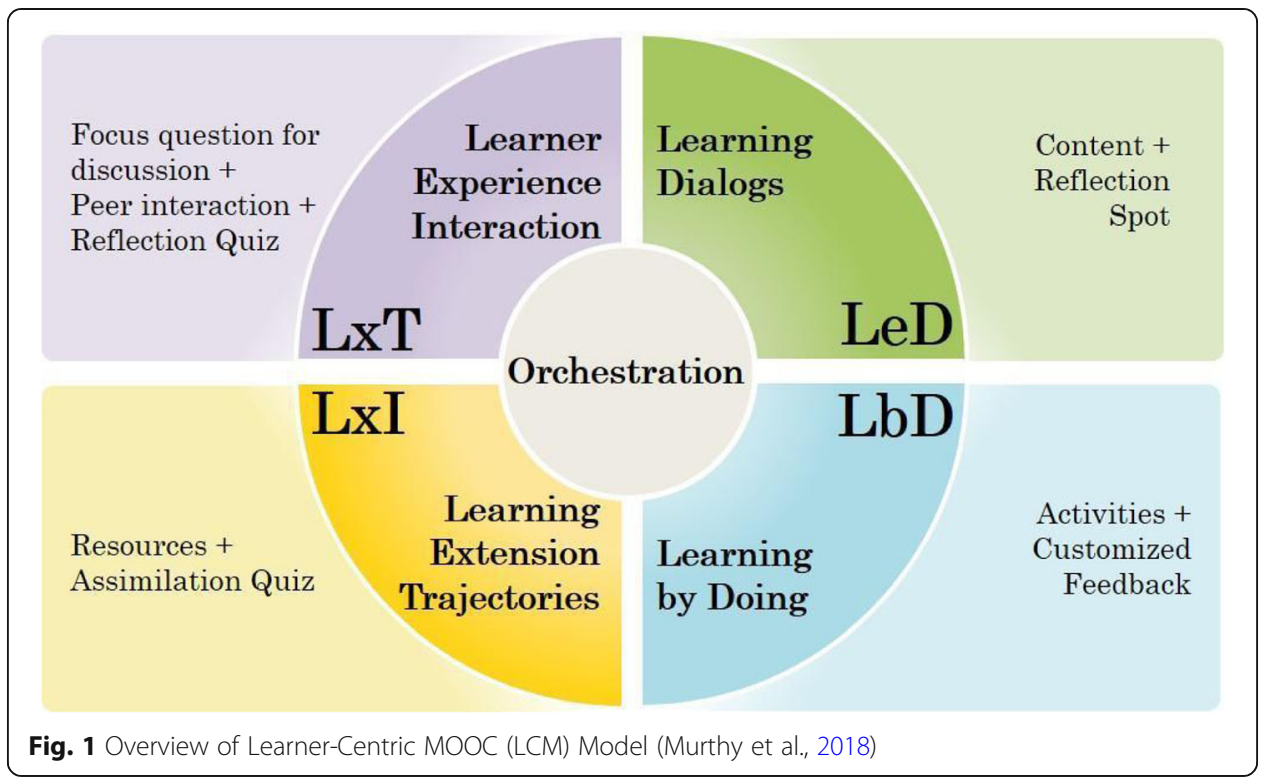

1. Learning dialogue (LeD): A short video with explicit reflection spots to promote concept attainment through learner interaction. It allows the learners to express prior conceptions, perform micro-practice or reflect. The instructor poses a multiple-choice question (MCQ) type question or activity, wherein, the students are expected to pause the video and respond to the question or activity.

2. Learning by Doing (LbD): Formative assessment activities with customized and constructive feedback.

3. Learning Extension Trajectories (LxT): advanced resource materials to diversify student's learning.

4. Learning Experience Interaction (LxI): a discussion forum activity, which cultivates the student interaction via structured discussion through the pre-set focus questions. The focus questions drive and keep the discussion centered on a specific topic, and ensures learner connect within MOOCs settings (Banerjee, Warriem, \& Mishra, 2018).

\section{Description of TEEL platform}

We orchestrated our physics course on the TEEL (Technology-enhanced and Evidencebased Education and Learning) platform (Ogata et al., 2018). It has five components, as shown in Fig. 2. The available literature report addresses a few of the challenges related to engagement and learning of students and strongly recommends using the learning analytics tools to evaluate the engagement and to support evidence-based learning. Extracting the learning log-data from the e-learning platform could then be helpful to redefine the instructional process and to provide feedback as suggested by Ogata et al. (2018).

The Learning Behavior Sensors captures the learner's and teacher's interaction data during a teaching-learning session. It offers MOODLE as the Learning Management System (LMS) and can integrate other e-learning tools. For instance, we used BookRoll (Ogata et al., 2015), an e-book reader. In BookRoll, the teachers share materials like their lecture slides and other reading material which the students can read and 


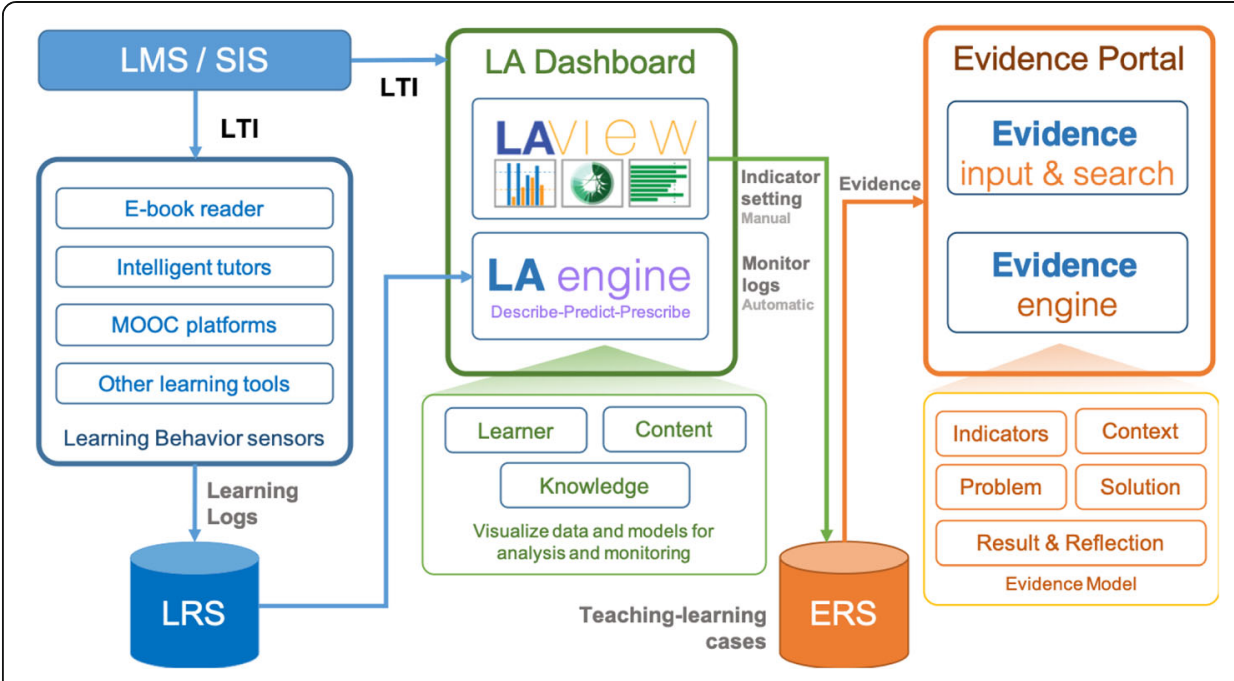

Fig. 2 Components of TEEL framework [Ogata et al., 2018]

annotate with doubts. BookRoll can automatically log the student's reading behavior and store it in the Learning Record Store (LRS). It is then available to both teachrs and students for analysis and reflection through the learning dashboard LAViEW (Majumdar, Akçapınar, Akçapınar, Flanagan, \& Ogata, 2019). LAViEW contains various panels of visualized indicators for monitoring and plays a central role to assist and identify problems in the teaching-learning scenario based on analysis of the visualized indicators. The other components are Evidence Record Store (ERS) and Evidence Portal. The Evidence Portal can be used to record the teaching-learning problem that the teacher identifies in LAViEW. The teacher can think of possible solutions to mitigate it and then monitor its effectiveness. Evidence Portal has an evidence analytics system which extracts evidence from LRS and metaanalyse evidence record in ERS. Thus the TEEL infrastructure integrates the features of the eReader, LMS and Dashboard within a single service so that teachers can seamlessly move across the technology. In the current study, we used the TEEL platform to orchestrate the F\&P strategy.

\section{Flip-phase - online component}

During the flip phase, we provided learner-centric activities as proposed in the LCM pedagogical model (as discussed in section II C). We divided each module of EPH 106 course into different sub-topics. For each topic, we created a full set of LCM learning activities. For example, Fig. 3 depicts the screenshot of flip-phase activity created for one of the topics, "X-ray diffraction" from the course module titled "Characterization techniques of Nanomaterials". An instructor can create their own LCM activities following the implementation steps as below.

- Provide Learning dialogue, LeD activity as short videos (less than $10 \mathrm{~min}$ ) for conceptual attainment. For example, we had provided four short lecture videos on the basic concepts of X-ray diffraction, Instrumentation details and working principle of X-ray diffractometer. Following the LeD videos, create a separate 'label' by using "Add an activity or resource" in the MOODLE and provide the reflection- 


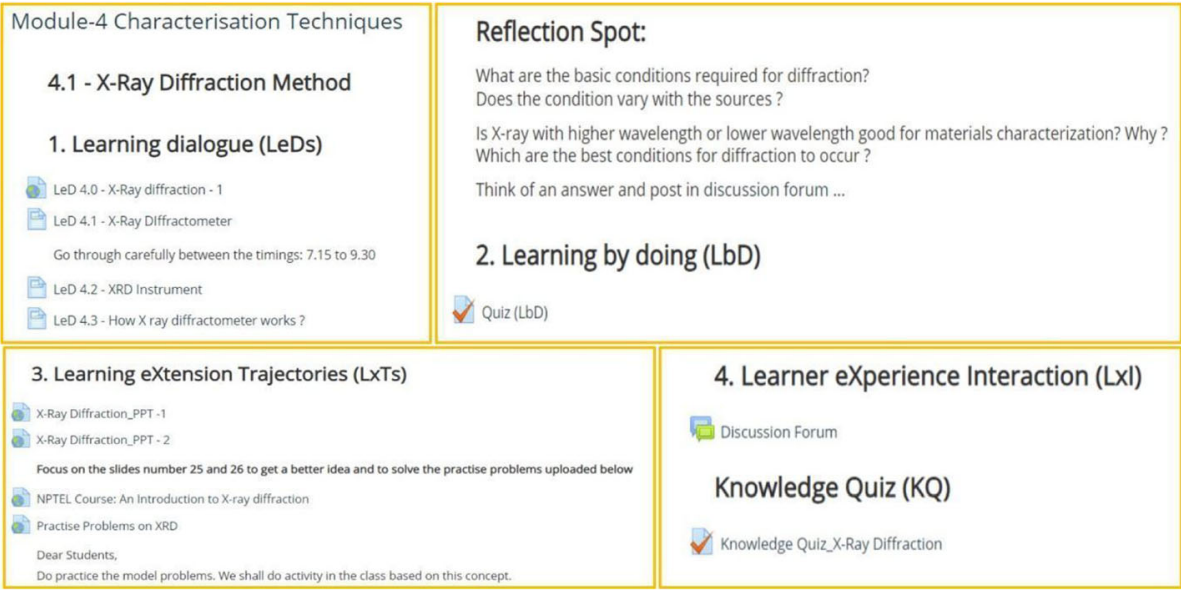

Fig. 3 Screenshot image of flip-phase activities for topic 'X-ray diffraction'

spot question for students to micro-practice their learning. Direct them to reflect upon their learning by posting their queries or comments in the forum activity.

- Upload Learning by doing (LbD) activity as a multiple choice question (MCQ) test with few conceptual questions. For example, in our EPH course, the questions for the LbD quiz were selected based on the concepts being taught in the LeD videos. To attempt the LbDs quiz, students took at least two or more attempts, as it was intended to be a formative assessment. We graded LbDs quiz automatically in the MOODLE.

- Provide Learning Extension Trajectories (LxT) activity, to diversify student learning. For example, in the present course, we uploaded the powerpoint presentations (.ppt) slides/notes, scanned book materials and solved problem sheets within the LxT activity. We additionally augmented a URL activity with the NPTel lecture notes (Alagarsamy, 2016) (Ray, 2017), Khan Academy or online simulator developed by "My Scope" (Microscopy, 2019).

- Provide Learner Experience Interaction (LxI) by creating a discussion forum activity in the MOODLE (Banerjee, Warriem, \& Mishra, 2018). Give a focus question to seed the discussions in the forum and to assist students to start a new discussion thread for the topic provided. For example, in our course, we created a LxI as the last activity as per the LCM learning sequence. We had given focus questions namely "Why do we observe peaks of different heights in the XRD pattern" to initiate the discussion thread on our target topic (as depicted in Fig. 4). The students then created and posted replies to the focus question. Upload the full set of LCM activities in the MOODLE, at least a week prior to the F2F sessions. Instruct students to go through the flipped contents before the F2F sessions.

\section{Pair-phase during F2F class component}

In the present course, we conducted the f2f sessions using an AL strategy, peer instruction (PI) for only selected topics following the $\mathrm{AB}$ type research design (details in section IV). We implement the PI activity with the following sequential steps: [1] Start the f2f teaching sessions first, by revisiting the flip phase learning contents as a short 


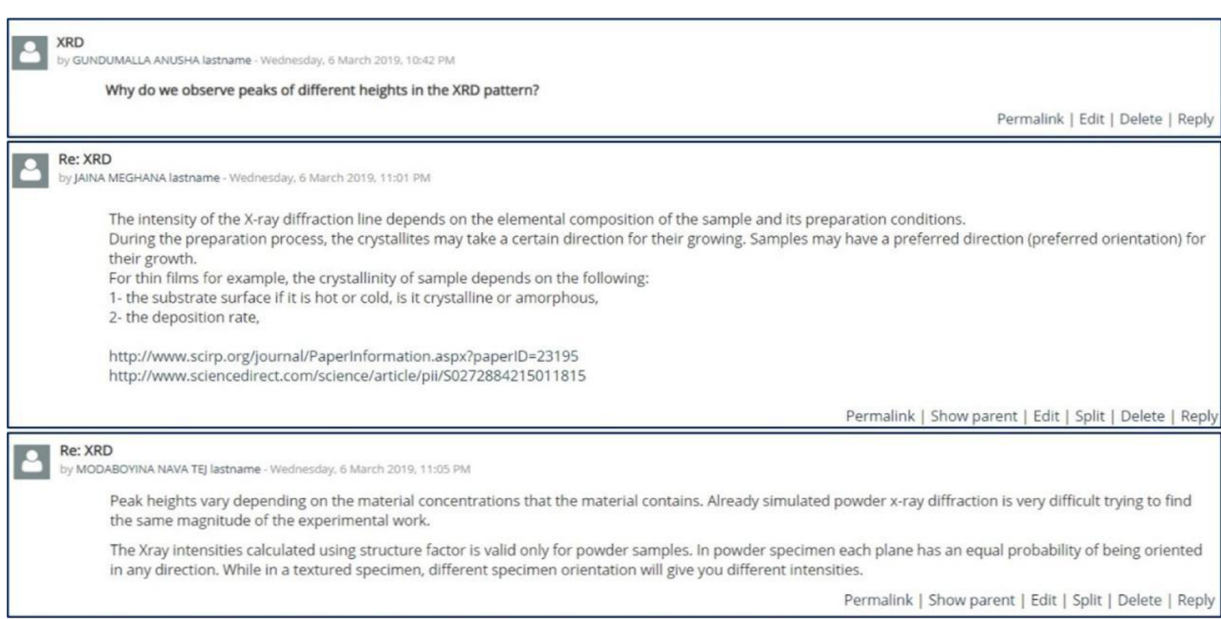

Fig. 4 Screenshot image of discussion forum $(L x \mid)$ activity with a focus question for topic dealt in F\&P method

lecture using blackboard or PowerPoint (.ppt) slides. Then, initiate the PI activity on concepts revised, by posing the PI question. [2] Instruct the students to answer the PI question first individually. If the majority of students answer incorrectly, ask the students to combine with 3 or 4 of their peers to form groups. [3] Initiate the pair-phase by allowing each group to discuss the PI question for about 10 to $12 \mathrm{~min}$. [4] During the pair phase, pose one or two hint questions that assist students to have a focused discussion and help them to arrive at the answers for PI questions. [5] Encourage students to have effective peer-peer discussions during the pair phase. Instruct the students not to deviate the discussion from the hint questions provided. [6] After the pairphase is over, pose the same PI question again to record the student responses. Check for improvement in the correct responses. Use the Plicker's card to collect student responses while conducting the PI activity during the f2f class (Hake, 1998). After the PI activity, assess the overall student performance of using knowledge quiz (KQ) as proposed in the LCM model. For example, in the EPH course, we conducted KQ either using the Plickers card or TEEL platform. We designed the quiz questions with MCQ, or True / False formats and graded them automatically in the MOODLE (Kothiyal, Majumdar, Murthy, \& Iyer, 2013).

\section{Research method and data collection}

The following are the overall research questions (RQs) addressed in the study.

RQ-1: What is the effect of F\&P strategy on student's engagement?

RQ-2: What is the effect of F\&P strategy on student's performance?

RQ-3: What are the student's perception of learning and engagement regarding the

F\&P strategy?

\section{Research design}

The materials provided to students in the flipped online component were designed by following the design of LCM pedagogical model (Murthy et al., 2018). We adopted the 


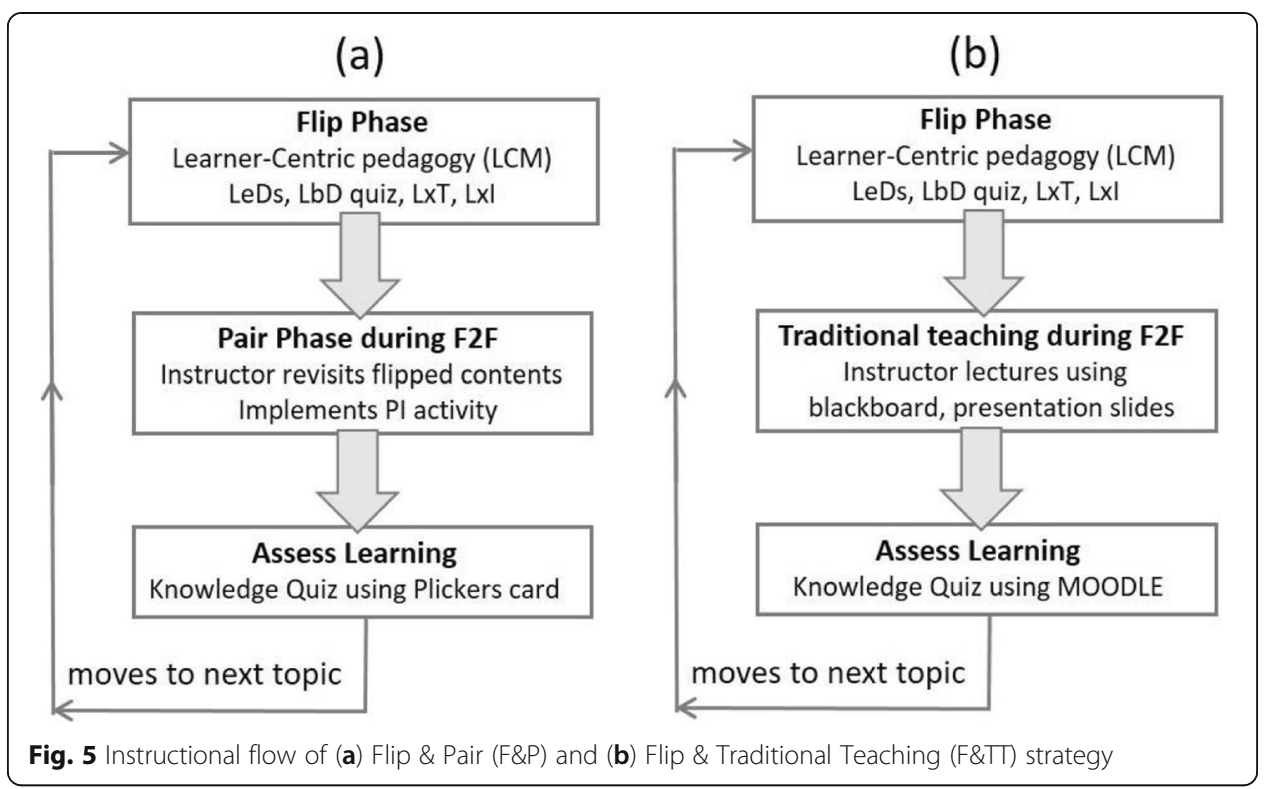

single group quasi-experimental AB-research design, wherein, the students were administered with two different teaching strategies in the blended physics course: (i) Type-A called as Flip \& Pair (F\&P) strategy, wherein, the LCM based learning activities for topic 1 was first flipped in TEEL platform for out-of-class learning. During f2f class, the same topic 1 was taught using the PI activity that included a 'pair phase' as discussed in Section III-B (Fig. 5a) and (ii) Type-B called as Flip \& Traditional Teaching (F\&TT), wherein, the LCM based learning activities for topic 2 was first flipped in the TEEL platform for out-of-class learning. During the f2f, the same topic was taught using traditional teaching (TT) without any collaborative learning activity (Fig. 5b).

Thus, both the type A and type B strategies had an AL pedagogy being included in the flip phase component, while it differed only in the teaching methodology adapted in the f2f class. This $A B$ research design was repeated for three rounds on multiple equivalent topics across the whole semester (topics dealt are shown in Table 1). However, in the present study, the data used to compare the effectiveness of PI are only from the third round of $\mathrm{AB}$ design implementation, while first two rounds were used to get the students adapted with the research design. The specific target topics and LCM learning objects from which, the student's MOODLE access report and the quiz performance scores were analyzed are listed in the Table 2. To check for the topics equivalence, we verified the following aspects: (i) learning time required, (ii) complexity level (iii) similarity in prerequisites of knowledge to learn the topics. We also triangulated the student perception about the equivalence and difficulty levels for both the target topics chosen. Care was taken to maintain the same duration of $f 2 \mathrm{f}$ class hours to teach the topics dealt for type-A and type-B interventions.

Table 1 Topics considered for AB-type research design

\begin{tabular}{ll}
\hline $\mathbf{F \& P}$ & F\&TT \\
\hline Giant Magnetoresistance & Nanotechnology-Significance and Challenges \\
Micro-Electromechanical Systems & Mechanical Properties \\
X-Ray Diffraction & Scanning Electron Microscopy \\
\hline
\end{tabular}

F\&P (type A) and F\&TT (Type B) strategies 
Table 2 Target materials used for the analysis for F\&P and F\&T strategy

\begin{tabular}{lll}
\hline LCM & Flip \& pair (F\&P) & Flip \& traditional teaching (F\&TT) \\
& Topic - X-ray diffraction (XRD) & Topic - scanning electron microscope (SEM) \\
\hline LeD & 1. Introduction to XRD & 1. Introduction to electron microscopes \\
& $\begin{array}{l}\text { 2. X-Ray diffractometer basics } \\
\text { 3. XRD Instrumentation }\end{array}$ & $\begin{array}{l}\text { 2. Electron Gun - Working Principle } \\
\text { 4. How X-Ray Diffractometer Works }\end{array}$ \\
& 3. Electron Gun - Thermionic Emission of electrons \\
LbD & Quiz on XRD & Quiz on Electron Gun \\
LXT & 1. XRD Lecture Slides & 1. SEM PDF material \\
& 2. NPTel Lecture Materials (Ray, 2017) & 2. NPTel Lecture notes for SEM (Alagarsamy, 2016) \\
3. Practice problems & 3. Electron Microscopy Book Materials \\
\hline
\end{tabular}

This study was implemented in freshman undergraduate engineering (B.Tech) students with the specialization (major) in electronics and communication. A total of 59 students were registered for the 'Physics of Nanomaterials' course (EPH 106) during the second semester. EPH 106 consisted of five modules and the same instructor taught the whole course. Each module was taught for a duration of twelve to fifteen 50-min lecture hours. The total duration to teach the course was sixty-five lecture hours for the whole semester. In the f2f component, few topics were taught via traditional teaching method and few of them using PI. Further, the learning contents were provided in the flip phase component, which used the TEEL MOODLE platform, wherein, every student was enrolled individually. The students were first briefed about the instructional design before the implementation. Table 3 presents the details of the data collected corresponding to the different constructs which are investigated in the research.

\section{Engagement analysis}

To address RQ-1, we analyzed the engagement of students in the content related to topics dealt in each of these strategies. We analysed the access frequency of the LCM learning contents provided for only the specific target topics (as listed in Table 2) to characterize the student engagement during the flip-phase activity in both the F\&P and F\&TT strategy. We counted the access frequencies to all the learning materials in the target topics orchestrated by F\&P and F\&TT method. Out of total 54 students, we used only $N=42$ student's log for this comparison as they were found to access the online LCM materials present in both the strategies.

Recently, Kothiyal et al. (2013) have developed and validated a 'Classroom Interactive Engagement Observation Protocol' an instrument to observe the student's behavior during the

Table 3 Data collected for different constructs in the study

\begin{tabular}{lll}
\hline Constructs & $\begin{array}{l}\text { Instruments } \\
\text { Engagement }\end{array}$ & $\begin{array}{l}\text { The data type (Number of students whose was data collected and } \\
\text { analyzed) }\end{array}$ \\
& $\begin{array}{l}\text { Lbservation } \\
\text { Protocol }\end{array}$ & $\begin{array}{l}\text { Access frequency, log data from TEEL Online Platform }(N=42) \\
4 \text { different 'Pair phase' of PI activity during F2F sessions }(N=52)\end{array}$ \\
Performance & $\begin{array}{l}\text { Knowledge Quiz } \\
(\text { KQ) }\end{array}$ & Post-Test score analysis for F\&P and F\&T $(N=44)$ \\
Perception & $\begin{array}{l}\text { Survey } \\
\text { Questionnaire } \\
\text { Personal Interview }\end{array}$ & Engagement, Learning, and Usefulness $(N=51)$ \\
\hline
\end{tabular}


'Think-Pair-Share (TPS)' activity in the f2f class. In this work, we had utilized those 'observation protocol items' to characterize the student engagement behavior during the 'Pair-phase' activity. Based on our raw observations of 14 different types of behaviors, we further classified them into three major engagement categories: actively engaged, passively engaged and not engaged. For example: talking to peers on topic, listening to peers, asking questions about the topic, group discussion, responding to teacher's questions are termed as 'actively engaged'. Whereas, reading or looking at one's own or neighbor's book, writing in a notebook, etc. are termed as 'passively engaged' and sleeping, talking off-topic, playing with mobile, sleeping head down, looking around the room and so on are termed at 'not engaged'.

\section{Performance analysis}

To address the RQ - 2, we compared the post-test mean scores obtained by students after the F\&P as against the F\&TT strategy. For the post-tests, the student's learning was evaluated for two different topics, for which the topic equivalence was checked as discussed in Section IV-A. Additionally, we implemented a similar type of KQ questions to maintain the same nature of testing for both the strategies. Though we adopted the two topics research design, we justify that both the post-tests are still comparable with each other. For the F\&P test there were scores of 44 learners and for the F\&TT, there were scores of 38 learners. Out of the cohort, 24 learners participated in both the tests. Based on the distribution of the score, as it did not satisfy normality condition (skewness $=-1.189997$ for F\&P and -0.785146 for F\&TT), we conducted a nonparametric Mann Whitney U test to evaluate the differences (see Table 4 for results).

\section{Perception analysis}

To answer the RQ-3, we collected the student perception on learning, engagement and usefulness. At the end of the course, an online survey questionnaire was provided to the students. It had 17 survey items ( 7 items related to engagement, 6 items related to learning and 4 related to usefulness) with a 5 Likert scale (refer to Tables - A1 to A3). It is to be noted that our students are in the first year engineering physics course and they experience the online flipped learning for the first time. Hence, the items in the survey questionnaire were designed mainly to understand the generic perception of students about the LCM learning activities in the flipped phase, that is present in common for both the F\&P and F\&TT strategies. However, one of the items (Item No - 7 in Tabe-1) was particularly related to the PI activity during the $\mathrm{f} 2 \mathrm{f}$ sessions. The responses for the perception questionnaire was collected and analyzed from a total number of students, $N=51$. Further, two open-ended questions were posed to collect the students' opinions on (i) using the online learning tools in the MOODLE platform and (ii) student's preferred sequence of access of LCM learning objects with respect to the instructor prescribed sequence: LeD - > LbD - > LxT - > LxI.

Table 4 Statistical difference analysis of the post-test after the F\&T and F\&P implementation

\begin{tabular}{llllc}
\hline Post test & N & $\begin{array}{l}\text { Median } \\
\text { (out of 10) }\end{array}$ & U & value* \\
\hline F\&TT & 38 & 5.29 & 1358.50 & 0.00 \\
F\&P & 44 & 7.81 & & \\
\hline
\end{tabular}

* Significant at $p$ value $<0.05$ 


\section{Results}

\section{Effects on students engagement}

\section{Online engagement for F\&P and F\&TT strategies}

The relationship between students' engagement and teaching strategies was shown in Fig. 6. It shows that there was more students' access frequency in F\&P strategy materials than F\&TT materials. The difference between the two strategies was statistically significant in LbD, LeD, and LxT $(p<.01)$. The difference was not statistically significant in LxI $(p=.90)$.

\section{In-class engagement during pair phase}

Figure 7 shows the analysis of the observation protocol data collected during the 'pair phase' of the PI activity. Our observation protocol data indicated that the nearly $25.9 \%$ of students being constructively discussing with their peers on the given task and nearly $19.20 \%$ were listening to the peers and finally, $18.20 \%$ were doing a group discussion (more than three) during peer instructional activities. Thus, we could observe that totally $67 \%$ of students displayed 'actively engaged' behavior, 18\% displayed 'passively engaged', and finally 15\% were 'not engaged' on an average for all four PI activities measured. The observation protocol data was further triangulated with the student's perception of engagement during the 'pair phase' of PI activity. The perception data showed that nearly $58 \%$ of students self-reported to be actively engaged, $35 \%$ reported being passively engaged, and 7\% not engaged during the activity.

\section{Effects on students' performance}

Table 4 highlights the difference of the post-test scores of students on the KQ conducted for different topics of F\&P and F\&TT strategies. A higher mean score $(M=7.81$ out of $10, S D=2.11$ ) was obtained for $F \& P$ as compared to that of the mean post-test

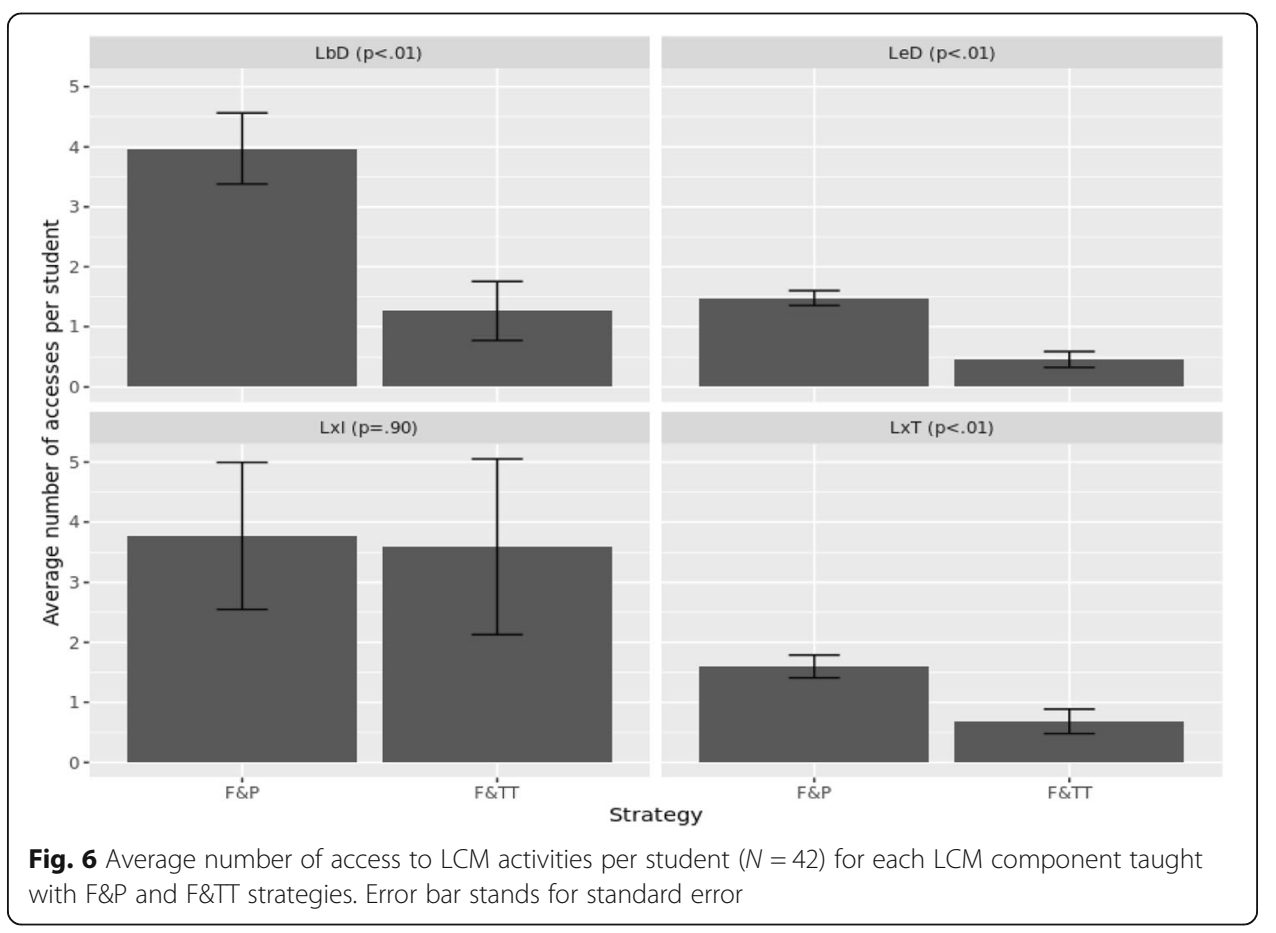




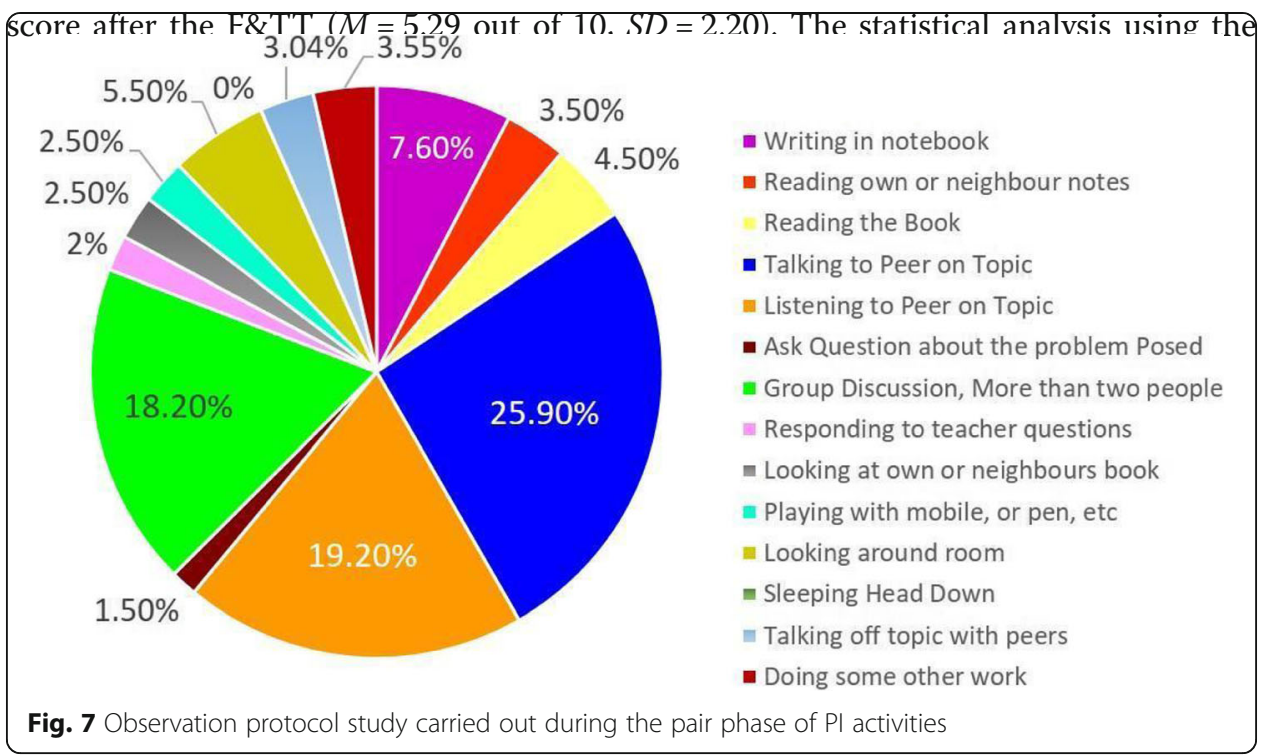

Mann Whitney $\mathrm{U}$ test between the post-test score showed that the differences were statistically significant $(\mathrm{U}=1358.5, p<0.05)$. Thus the $\mathrm{KQ}$ post-test score analysis inferred that the F\&P had better learning effectiveness when compared to the F\&TT type of intervention. The observed effect size is 0.54 which can be considered as a moderate treatment effect. When the analysis was repeated only for paired sample $(n=24)$, performance during the F\&P knowledge quiz still remained significantly better than F\&TT quiz $(\mathrm{U}=116.5, p=0.00013)$ with moderate effect size.

\section{Student's perception analysis}

The RQ-3 was answered by corroborating the student perception of engagement with that of engagement results obtained from the access log data in the TEEL platform Fig. 8a. The student's self-reported average engagement time in the flip phase activities is shown in the Fig. 8b. We observed that the students showed a high perception of engagement for all the LCM activities including the LeDs, LbDs and LxT resources. However, the students were found to be less encouraged to participate in in the LxI discussion forum activity was found to be. Fig. 9 shows that a considerable number of students had a high perception of learning from the LeDs (72\%) and LbDs (74\%) activities. Nearly $73 \%$ of students reported that the LxT activities helped them to diversify their learning. In contrast to other LCM elements, only $65 \%$ of students perceived the LxI forum as an important component of learning. Student's perception of engagement in the flip activities showed a high level of satisfaction for both LeDs and LxT resources, while engagement in discussion forum activity was perceived to be low as shown in Fig. 9. The student preferred to get engaged in following access sequence: LeDs $(44 \%) \rightarrow$ LxTs $(37 \%) \rightarrow$ LbDs $(13 \%) \rightarrow$ LxIs $(6 \%)$.The students considered a high perception of usefulness for the F\&P activities as shown in Fig. 10, where 67\% of the students agreed that they will be happy if the other teachers would adopt the F\&P teaching strategy for their courses. When the students were given choices to opt for the self-estimated total time spent per day on the LCM activities, nearly $49 \%$ of 


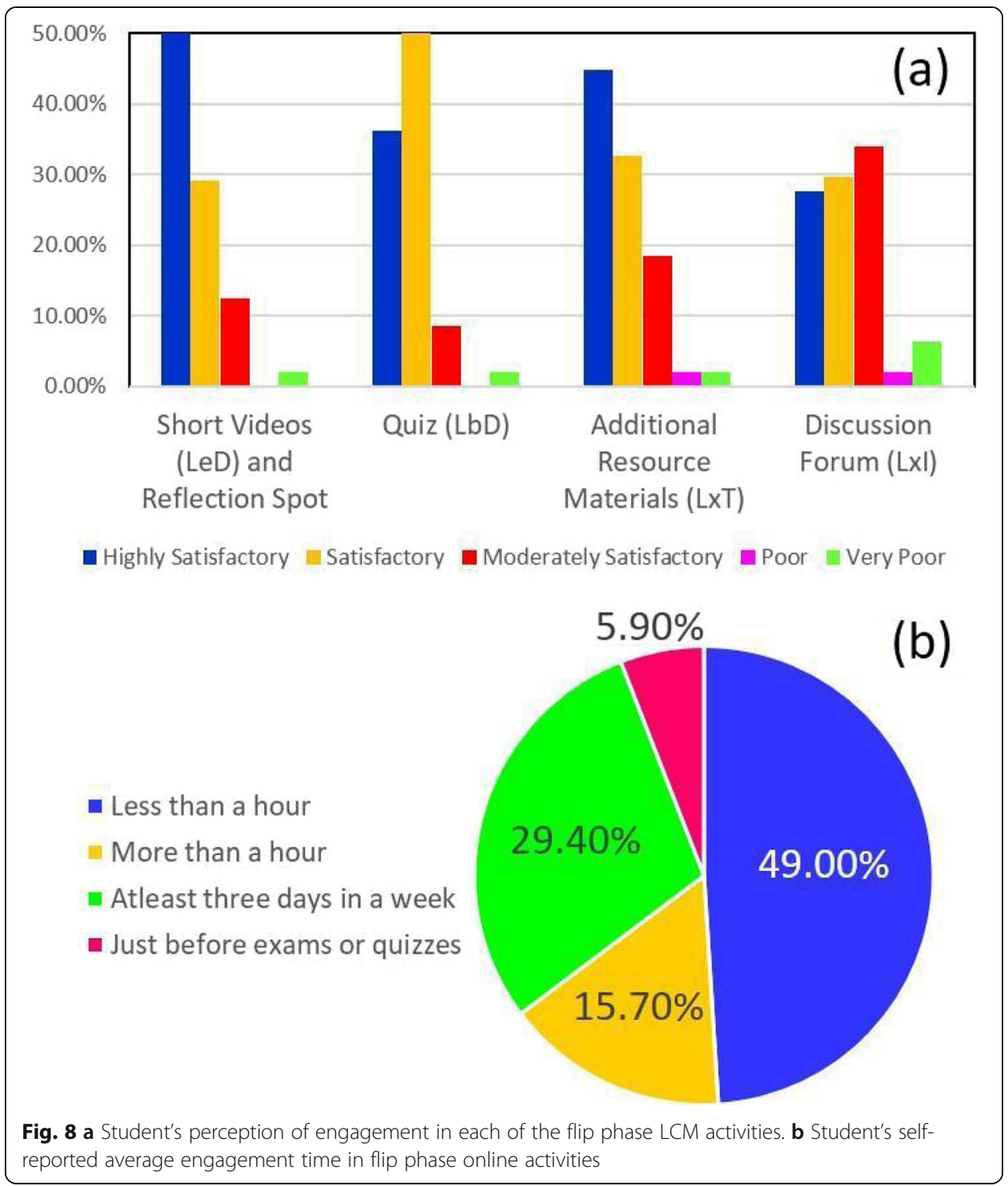

students agreed for 1 hour/day, 15.7\% for more than 1 hour/day, $29.40 \%$ for at least 3 days/week, 5.9\% for just before mid/semester exams (Fig. 9 b).

\section{Reflections from the instructor}

Presently, we adapt the LCM model to design the learning activities of flip phase as follows: LeDs as videos $\rightarrow$ LbDs as Quizzes $\rightarrow$ learning resources as LxTs $\rightarrow$ discussion forum as LxIs. From the log data report as obtained from LAView analytics, we inferred a poor engagement of students in the flipped activities during the first few weeks of implementation, though we orchestrated them with learner-centric activities. This observation is in accordance with the case study reported by $\mathrm{Li}, 2018$ ( $\mathrm{Li}, 2018)$, which highlights the possible reasons as (i) student's inadaptability to the e-learning platform, (ii) instructional design or (iii) teacher's inadequate knowledge of the prerequisite practice required. In the present study, integrating a learner-centric pedagogy, the F\&P research design within the BL course is a first attempt the instructor has made for an 


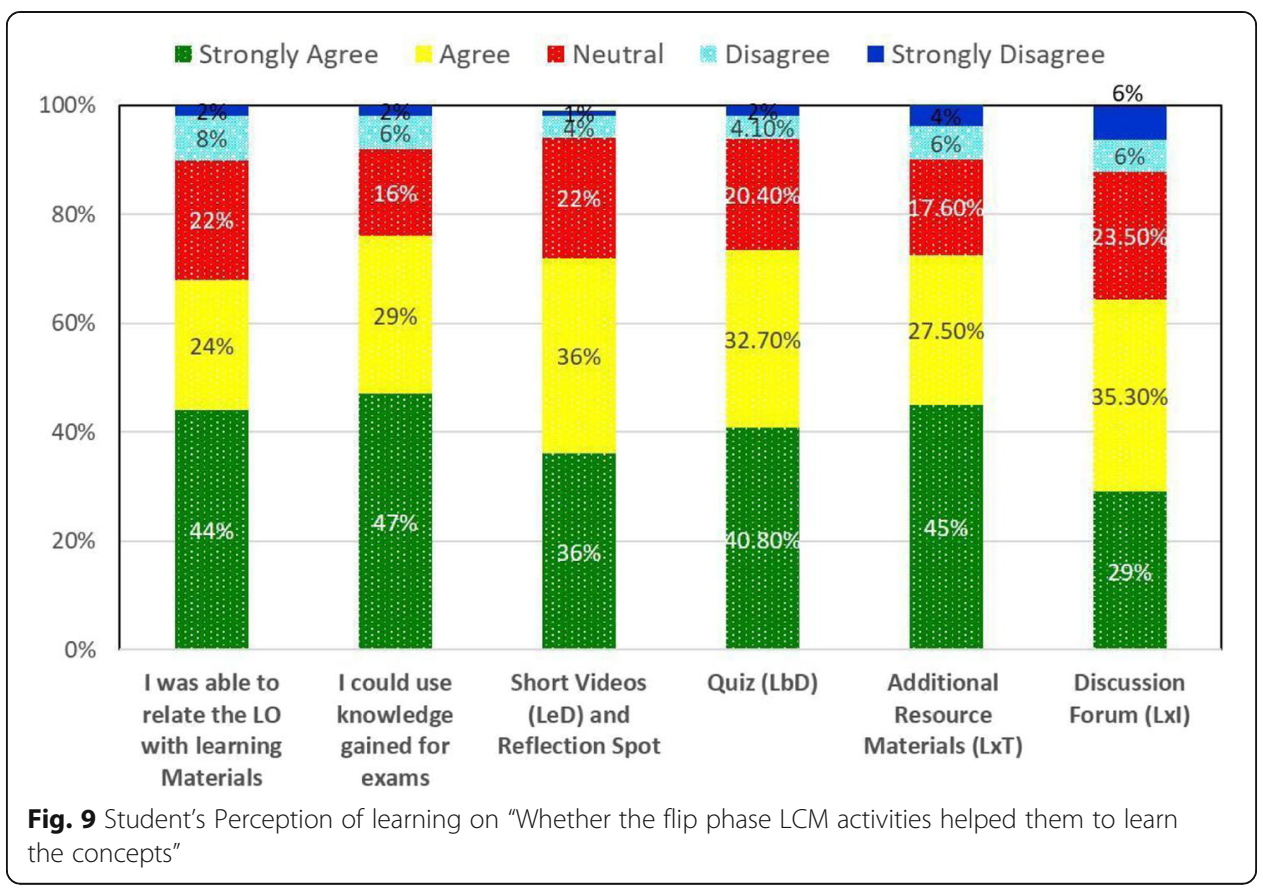

undergraduate engineering physics course. Thus, we had primed the students with this new instructional design until they got familiarized with the instructional design. It also facilitated the instructor to improvise the design with respect to the present teaching context and also to get appropriate control of implementation.

Additionally, to improve the engagement in the TEEL platform, few key measures were prompted during the f2f sessions. This step further improved the online engagement of students considerably. The measures taken were (i) augmenting the LxTs with NPTel videos and lecture notes (SantiramKal, 2004), (ii) incentivize those students who engage in flip phase activities, (iii) group activities in LxI to post, create or reply in discussion forum activity, (iv) counselling students who showed poor engagement and

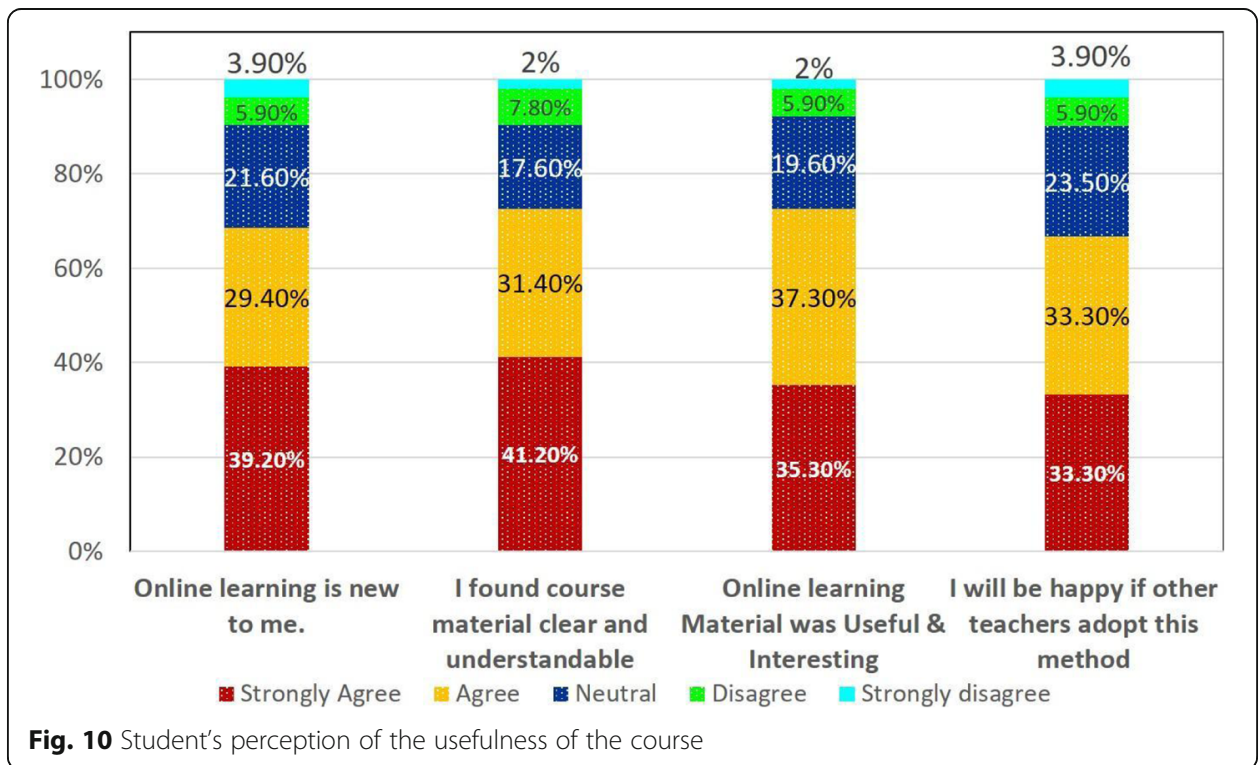


performance. These students were provided with required extra learning materials to bridge the lack of fundamental knowledge, Prior to the mid- or end-semester examinations, the important learning resources were provided within the LxTs activity. This allowed many students to revisit the learning objects in MOODLE multiple times, thereby increasing the access frequency of the log data. Finally, (v) the students were asked to practice the theoretical concepts being taught for the target topics of electron microscopy and X-ray diffraction using the open-source online simulations developed by 'My Scope ${ }^{\text {two }}$ (Microscopy, 2019). MyScope ${ }^{\text {Tw }}$ is an online training tool to learn different types of scanning probe and diffraction techniques. The learners were able to virtually operate these high-end machines to get a realistic operational experience. Students had the freedom to change the working mode of operation, vacuum conditions and different type of samples. Other parameters could be varied such as the accelerating voltage, beam spot size, scan speed and the working distances to achieve the required magnification and resolution of the final imaging. The step-by-step instructional guide with appropriate background knowledge, diagrams and many other extended learning web-resources provided within the MyScope website facilitated the students to explore and learn individually. These online simulators helped the students to improve the active engagement capacity through the kinesthetic learning. More importantly, the students felt it safe to make mistakes without any fear while operating these machines virtually.

\section{Discussion and conclusions}

Inferences from the study

The research design adopted in our current study aims to compare the F\&P versus F\&TT strategies with the $\mathrm{AB}$ research design. In both the types, the online flip phase contents were augmented with learning activities based on pedagogical design of the LCM. It is to be noted that these two strategies differed only in the teaching method adapted during the $\mathrm{f} 2 \mathrm{f}$ class, wherein, the F\&P had an active PI-based learning activities while it was the traditional teaching for F\&TT. We had totally implemented three rounds of the AB research design for equivalent topics (as listed in Table-1). However, according to the instructor's observation, the students did not understand the flipped classroom method during the initial few weeks of implementation. Hence, the first two rounds of AB design were used to allow the students, to get adapted to the flipped classroom method, tools of TEEL (LMS) platform and the learning activities based on the LCM pedagogy.

To evaluate the effectiveness of research design, first, we extracted and analyzed the student access frequency for target materials (as listed in Table 2) provided in the flip phase for both the F\&P and F\&TT strategies. The overall access frequency extracted for the learning activities across the whole semester inferred that the average number of log access was higher for the contents taught with the F\&P strategy when compared to the F\&TT (Fig. 6). The higher log access frequency in the flip phase activities for both teaching strategies infer that the students were able to effectively utilize and learn from the LCM online learning materials throughout the semester. Our present flippedlearning course can be corroborated with the research highlights by Brame et al. (Brame, 2015) on the higher student learning engagement achieved from interactive online videos. In accordance to their reports, we observed that the reflection spots provided in the LeD videos had facilitated the students engagement, thus enabling higher 
learning to take place. This could be further evidenced from the new discussion threads created by the students in the LxI forum activity attempting to answer the reflection spot questions. Similarly, the LxTs with diverse resources from the NPTel lecture notes has helped students to attain conceptual acquisition and supported the extended learning.

The student's engagement behavior during the pair-phase activity of F\&P strategy was characterized using the observation protocol items (Kothiyal et al., 2013). This study indicated that the majority of students exhibited 'actively engaged' behavior during the Pair-phase of the PI activity. As pointed out by Hamdan et al. (Hamdan et al., 2013), the flipped classroom strategy demands the students to engage in flip contents before the f2f sessions, and during the class they need to perform task-oriented higherorder activities on the same contents under the facilitation of instructor. However, in contrast, with respect to the F\&TT (Type-B intervention), the students mostly exhibited passive listening during the f2f traditional lecturing sessions. The students were neither concerned to raise their doubts nor responded to any interactive learning efforts made by the instructor. Our study supports different existing literature on the flipped teaching implemented for multiple teaching-learning contexts that the traditional teaching leads to the passive listening of students (Goodwin \& Miller, 2013). Few of our students did not get engaged in the flipped activities due to the low motivation towards the online learning and other logistic issues such as the poor internet connectivity, etc. Hence, in our present teaching-learning context, the instructor had to revisit the flip-phase contents again during the $\mathrm{f} 2 \mathrm{f}$ sessions.

While comparing the access frequencies between F\&P and F\&TT strategies, we observed statistically significant differences in the access frequency for all the LCM learning components except for LxI forum activity. The incentives provided for students participating in LxI forum may be the plausible reason for a lack of significant difference between access frequencies in both the strategies. However, the same effect is not observed for other LCM components. In the current scenario, we indicate that comparing the access frequency between these two strategies is still valid as we adopted similar flip phase learning design for both F\&P and F\&TT type of interventions. To justify our results on higher access frequency for the F\&P intervention, we decipher that the contents dealt with active-learning PI pedagogy during the f2f class sessions has helped the students to engage and learn those content with more clarity. The peer-peer collaborative learning activities has fostered the student's interests to enhance their motivation to revisit the same learning materials provided in the flip phase, even after the f2f sessions were over. We infer that this might be one of the reasons to enhance the access frequency for F\&P type of intervention. Furthermore, the access frequency data was reliable because the results were consistent over three components in the LCM model (LeD, LbD and LxT) except for LxI. The performance analysis using paired t-test on the post-test scores of F\&P and F\&TT revealed a statistically significant $p$-value $(p<$ 0.05 ) and better mean score as compared to F\&TT. We also infer that F\&P method contributed better to achieve the higher quiz score more than F\&TT. We show that improved engagement during the F\&P strategy has positively correlated with the student performances. The increase in the mean score for F\&P showed that the teaching using the collaborative learning strategy PI within f2f settings had led to the better conceptual learning of materials. Students being engaged both during Pair phase and the flip phase activities in case of the F\&P strategy, emerges to be an important factor to 
explain the improved performance and satisfaction of students. Our observation supports the literature that reports an engagement stimulated by flipped learning pedagogy is inherently satisfying to students independent of their perceptions of performance. (Fisher, Birdthistle, \& Perényi, 2018).

We further obtained the student perception on the F\&P blended course. We conducted an unstructured interview with 8 students selected based on the low, medium and high performers. We obtained the student's reflections on three open-ended questions. The first question assessed how did the F\&P strategy help to enhance learning of physics concepts, and whether the strategy can be adapted as a method of active instruction by other instructors?. The second question investigated on what kind of challenges the students faced while using the new learning tools of TEEL platform? . Finally, the third question was on which of the LCM learning activity was useful for the conceptual attainment while considering flipped classroom? . Regarding the first question, students expressed that each of LCM activity was unique and helped them to better understand the physics concepts. Irrespective of performance, the students who were interviewed told that this new flipped teaching motivated their interest to learn physics subject as a full course. They expressed that they could find all the relevant learning materials at one place (MOODLE) to read them before any upcoming mid- or end-semester exams. Additionally, the students were quite motivated to learn through peer-peer discussions in the f2f class. Some students felt it difficult to understand the format of PI activities initially but later-on found it very interactive. Additionally, they expressed that providing the answers for PI questions using the Plicker cards made the PI activity more interesting and engaging. Some students felt that there were noisy disturbances from other students during the PI activity that led them difficult to concentrate on the tasks given. Thus, the perception survey analysis showed that a larger percentage of students exhibited positive perception of learning from F\&P. They could utilize the effectiveness of AL pedagogies of both the LCM and PI. Nevertheless, few of the students whom we identified as poor performers seemed to have prejudice that physics courses would be boring. These students also expressed that they had never been interested to learn the subject with a better conceptual clarity. They just studied physics only to pass the examination even during their school education. It seemed to be crucial to change this low perception of learning basic science courses at this juncture of firstyear engineering, which is only possible by adapting AL pedagogies. With regard to the second question on challenges while handling the technology tools, most of the students expressed that the BookRoll, an ebook reader as provided in the TEEL platform was new to them and it was quite useful to annotate their doubts. However, they required more mobile-friendly features and better training to handle the same. There were many other challenges such as possessing individual laptops and gadgets, time restrictions in internet availability for hostel students. Further, when alternated with their mobile data connection to attempt MOODLE quizzes, they were facing low connectivity issues.

The student response to the third question pointed out that most of them agreed to the fact that learning from the LeD videos and LxT resources were quite helpful. They got benefited from being able to revisit the videos and explore the learning materials multiple times at their own pace. Majority of students mentioned that the LxI posts/replies are not getting diverse responses. Additionally, there were lack of coherence in a LxI replies which reduced the overall motivation of students. Few students also 
reported that they were not clear about the operating aspects of MOODLE, for example, 'reply to', 'posts', etc. of LxI forum. The LxI activity allowed the students and the teachers to exchange ideas by posting queries/doubts, as part of a 'discussion thread'. Thus, the role of an instructor is crucial to provide clear directions to guide and moderate the LxI activity along with a timely feedback. The student perception analysis could be correlated with that of the instructor's observation on the discussion forum activities. We found that the quality of post created was not of a good standard and students just copied the contents from Google or Wikipedia. The discussion threads did not show any coherent replies beyond 3 or 4 threads. However, few of the good performers gave thoughtful answers and replied to the queries raised in their peers' post. The quality of reply, regular engagement of poor performers in the LxI forum was not very evident. It is also correlated with that of the poor perception exhibited for learning from LxIs. We infer that the significant relation between the engagement of students in the forum activity with that of the performance score could be less prominent.

From the present study, we show that the F\&P strategy could be effectively integrated into the flipped classroom for undergraduate engineering students. We recommend that the students and teachers need to be primed to get adapted with the instructional strategy and technology tools in MOODLE. Further, integrating the LCM based pedagogical activities such as the LeDs as the short videos, Quizzes as LbDs, LxTs as resource materials, a discussion forum as the LxIs and the knowledge quizzes are necessary to create a meaningful learning experience in the flip phase.

\section{Limitations and future work}

We identify a few limitations while implementing the present research design. To bring-in the contextual improvisations, we had deviated from the originally proposed LCM model with respect to the orchestration of learner-centric activities (Murthy et al., 2017; Murthy et al., 2018). For example, we did not provide the customized feedback for LbD quiz as instructed in the LCM model. Then, the reflection spots were not embedded within the LeD videos. Instead, it was provided as a separate label in the MOODLE course page. Further, the assimilation quiz or reflection quiz was not adapted. This was because, the instructor is a novice practitioners of educational research and took time to get acquainted with the new features of TEEL tool and the operational aspects of the MOODLE. Others limitations are in the methods of evaluation. The $A B$ research design is a single group post-test analysis limited to the test score for only two target topics. Thus, $\mathrm{AB}$ design within the blended course needs to be tested for multiple topics having more number of baseline and treatment data points.

We measured the quantitative engagement data using the learning log data in LMS. Though there is an indication that key measures have enhanced the frequency of log access counts, in the LCM activities, we could not adequately create instances for cognitive engagement within the online content. Thus, it is highly desirable to use more effective tools to collect real-time evidence of engagement and learning. Furthermore, the indicator we used, i.e., access count in MOODLE for each LCM component is considered as a proxy for student engagement. Hence other indicators such as 'time duration', or 'type of engagement in activity' will be used for future studies. Lastly, we consider the F\&P strategy was implemented and studied in a very specific socio-cultural context and for a particular subject course. Future studies should aim to investigate its generalizability in another context. 


\section{Abbreviations}

F\&P: Flip and Pair; ERS: Evidence Record Store; KQ: Knowledge Quiz; LbD: Learning by Doing; LCM: Learner-Centric MOOCs; LeD: Learning dialogue; LMS: Learning Management System; LRS: Learning Record Store; LXT: Learning Extension Trajectories; Lxl: Learning Experience Interaction; MCQ: Multiple Choice Questions; OER: Open Educational Resources; PI: Peer Instruction; TEEL: Technology-enabled and Evidence-based Education and Learning

\section{Acknowledgements}

The authors would like to thank Prof Sahana Murthy, Indian Institute of Technology (IIT) Bombay for the support. The authors acknowledge the B. Tech (ECE - Section II) students of 2018-19 batch at GITAM Hyderabad for their cooperation in conducting this research.

\section{Competing of interest}

The author has no competing interests.

\section{Authors' contributions}

Portions of these findings were presented as a full Paper at the 2019 IEEE Tenth International Conference on Technology for Education (T4E), 2019. We have no conflicts of interest to disclose. The authors read and approved the final manuscript

\section{Funding}

This work was partly supported by NEDO Special Innovation Program on AI \& Big Data 18102059-0, JSPS KAKENHI Grant-in-Aid for Scientific Research (S) Grant Number $16 \mathrm{H} 06304$ and JSPS KAKENHI Research Activity Start-up Grant Number $18 \mathrm{H} 05746$

\section{Availability of data and materials}

Not Applicable.

\section{Author details}

${ }^{1}$ Department of Physics, GITAM School of Sciences, GITAM (Deemed to be University), Rudraram, Hyderabad 502 325, India. ${ }^{2}$ Graduate School of Informatics, Kyoto University, Kyoto, Japan. ${ }^{3}$ LET Research Unit ACCMS, Kyoto University, Kyoto, Japan. ${ }^{4}$ NPTEL, Indian Institute of Technology Madras, Chennai, India.

\section{Received: 22 April 2020 Accepted: 6 September 2020}

Published online: 27 October 2020

\section{References}

Alagarsamy, I. P. (2016). Characterization of materials Retrieved from NPTEL: https://nptel.ac.in/courses/115103030/. Aronson, E., \& Patnoe, S. (1997). Cooperation in the classroom: The jigsaw method. New York: Longman. Bach, S. (2007). Online learning and teaching in higher education. New York: Mc Graw Hill, Open University Press.

Banerjee, G., Warriem, J., \& Mishra, S. (2018). Learning experience interaction (Lxl): Pedagogy for peer-connect in MOOCs. In Proceedings of the 26th international conference on computers in education (pp. 715-724). Philippines: Asia-Pacific Society for Computers in Education.

Barron, B. J. S., Schwartz, D. L., Vye, N. J., Moore, A., Petrosino, A., et al. (1998). Doing with understanding: Lessons from research on problem- and project-based learning. The Journal of the Learning Sciences, 7, 271-311.

Beer, C., Clark, K., \& Jones, D. (2010). Indicators of engagement. In Proceedings of ASCILITE - Australian society for computers in learning in tertiary education annual conference, (pp. 75-86).

Bernard, R. M., Borokhovski, E., Schmid, R. F., Tamim, R. M., \& Abrami, P. C. (2014). A meta-analysis of blended learning and technology use in higher education: From the general to the applied. Journal of Computing in Higher Education, 26(1), 87-122.

Bonwell, C. C. (1996). Enhancing the lecture: Revitalizing a traditional format. In T. E. Sutherland, \& C. C. Bonwell (Eds.), Using active learning in college classes: A range of options for faculty, (pp. 31-44). San Francisco: Jossey-Bass.

Brame, C. (2015). Effective educational videos Retrieved March 14, 2020, from https://cft.vanderbilt.edu/guides-sub-pages/ effective-educational-videos/.

Brusilovsky, P. (2003). Adaptive and intelligent web-based educational systems. International Journal of Artificial Intelligence in Education, 13(2-4), 159-172.

Buket, A., \& Meryem, Y. S. (2008). A study of student's perceptions in a blended learning environment based on different learning styles. Educational Technology \& Society, 11, 183-193.

Chen, Z., Stelzer, T., \& Gladding, G. (2010). Using multimedia modules to better prepare students for introductory physics lecture. Physical Review Special Topics - Physics Education Research, 6, 108

Chew, C., \& Wee, L. K. (2015). Use of blended approach in the learning of electromagnetic induction arXiv:1501.01527v2 [physics.Ed-ph]

Crouch, C. H., \& Mazur, E. (2001). Peer Instruction: Ten years of experience and results. American Journal of Physics, 69, 970, https://doi.org/10.1119/1.1374249.

Erhan, E. (2016). "Why do I slog through the physics?" understanding high school students' difficulties in learning physics. Journal of Education and Practice, 7(7), 95-107.

Ferreira, H., de Oliveira, G., \& Araújo, R. (2019). Technology-enhanced assessment visualization for smart learning environments. Smart Learning Environment, 6, 14. https://doi.org/10.1186/s40561-019-0096-z.

Fisher, R., Birdthistle, N., \& Perényi, A. (2018). The positive relationship between flipped and blended learning and student engagement, performance and satisfaction. In Active Learning in Higher Education, (pp. 1-17).

Garrison, D. R., \& Vaughan, N. D. (2008). Blended learning in higher education framework, principles, and guidelines. Hoboken: A Wiley Imprint.

Glazer, F. S. (2011). Blended learning: Across the disciplines, across the academy. New pedagogies and practices for teaching in higher education. Sterling: Stylus Publishing, LLC. 
Goodwin, B., \& Miller, K. (2013). Evidence on flipped classrooms is still coming in. Educational Leadership, 70(6), 78-80.

Guo, P. J., Kim, J., \& Robin, R. (2014). How video production affects student engagement: An empirical study of MOOC videos. In ACM conference on learning at scale (L@S 2014).

Hake, R. R. (1998). Interactive-engagement versus traditional methods: A six-thousand-student survey of mechanics test data for introductory physics courses. American Journal of Physics, 66(1), 64-74. https://doi.org/10.1119/1.18809.

Halverson, L. R. (2016). Conceptualizing blended learning engagementTheses and dissertations, (p. 5981).

Hamdan, N., McKnight, P., McKnight, K., \& Arfstrom, K. M. (2013). The flipped learning model: A white paper based on the literature review titled "a review of flipped learning". Arlington: Flipped Learning Network.

Henrie, C., Halverson, L., \& Graham, C. (2015). Measuring student engagement in technology-mediated learning: A review. Computers in Education, 90, 36-53. https://doi.org/10.1016/j.compedu.2015.09.005.

Hrastinski, S. (2019). What do we mean by blended learning? Technical Trends, 63(5), 564-569.

Hsin, W. J., \& Cigas, J. (2013). Short videos improve student learning in online education. Journal of Computing Sciences in Colleges, 28, 253-259.

Imran, H. (2019). Evaluation of awarding badges on student's engagement in Gamified e-learning systems. Smart Learning Environments, 6, 17. https://doi.org/10.1186/s40561-019-0093-2.

Johnson, A. M., Jacovina, M. E., Russell, D. E., \& Soto, C. M. (2016). Challenges and solutions when using technologies in the classroom. In S. A. Crossley, \& D. S. McNamara (Eds.), Adaptive educational technologies for literacy instruction, (pp. 13-29).

Kannan, V., \& Gouripeddi, S. P. (2018). Enhancement in critical thinking skills using the peer instruction methodology. In The 18th IEEE international conference on advanced learning technologies, (p. 307). https://doi.org/10.1109/ICALT.2018.00127.

Kannan, V., \& Gouripeddi, S. P. (2019). Contextualising the learner-centric MOOCs model for effective blending of flippedclassroom method in engineering physics course, (p. 46). Goa: 2019 IEEE Tenth International Conference on Technology for Education (T4E). https://doi.org/10.1109/T4E.2019.00-51.

Kay, R. (2011). Evaluating learning, design, and engagement in web-based learning tools (WBLTs): The WBLT evaluation scale. Computers in Human Behavior, 27(5), 1849-1856. https://doi.org/10.1016/j.chb.2011.04.007.

Kim, J., Guo, P. J., Seaton, D. T., Mitros, P., Gajos, K. Z., \& Miller, R. C. (2014). Understanding in-video dropouts and interaction peaks in online lecture videos. In Proceedings of the first ACM conference on learning @ scale confereAssociation for computing machinery, (pp. 31-40). New York. https://doi.org/10.1145/2556325.2566237.

Kothiyal, A., Majumdar, R., Murthy, S., \& lyer, S. (2013). Effect of think-pair-share in a large CS1 class: 83\% sustained engagement. In ICER '13: Proceedings of the ninth annual international ACM conference on international computing education research, (pp. 137-144). https://doi.org/10.1145/2493394.2493408.

Kuromiya, H., Majumdar, R., Warriem, J., \& Ogata, H. (2019). Data-driven validation of pedagogical model - a case of blended LCM model, (pp. 38-45). Chennai: IEEE Tenth International Conference on Technology for Education (T4E). https://doi.org/ 10.1109/T4E.2019.00016.

Li, Y. (2018). Current problems with the prerequisites for flipped classroom teaching-a case study in a university in Northwest China. Smart Learning Environments, 5(2), 1-23. https://doi.org/10.1186/s40561-018-0051-4.

Lin, S.-Y., Aiken, J. M., Seaton, D. T., Douglas, S. S., Greco, E. F., Thoms, B. D., \& Schatz, M. F. (2017). Exploring physics students' engagement with online instructional videos in an introductory mechanics course. Physical Review Physics Education Research, 13, 020138.

Linghong, L., \& Tang, H. T. (2017). Teaching physics with blended learning. Journal of Modern Education Review, 7, 231. https:// doi.org/10.15341/jmer(2155-7993)/04.07.2017/001.

Majumdar, R., Akçapınar, A., Akçapınar, G., Flanagan, B., \& Ogata, H. (2019). LAView: Learning analytics dashboard towards evidence-based education. Tempe: The 9th International Conference on Learning Analytics \& Knowledge (LAK19).

McTighe, J., \& Lyman, J. (1988). Cueing thinking in the classroom: The promise of theory-embedded tools. Educational Leadership, 45(7), 18.

Meltzer, D. E., \& Thornton, R. K. (2012). Resource Letter ALIP-1: Active-Learning Instruction in Physics. American Journal of Physics, 80, 478. https://doi.org/10.1119/1.3678299.

Michele, W. M., Rose, A. F., George, H., \& Zuleyha, Y. (2020). PERC proceedings edited by wolf, Bennett, and frank; peer-reviewed, American Association of Physics Teachers. https://doi.org/10.1119/perc.

Microscopy, A (2019). Scanning probe \& atomic force microscopy Retrieved 2018, from Mlcroscopy Australia: https://myscope. training/.

Mintzes, J. J., \& Walter, E. (2020). Active learning in college science - the case for evidence-based practice. Switzerland: Springer Nature.

Murthy, S., Warriem, J., \& lyer, S. (2017). Technology integration for student-centered learning: A model for teacher professional development programs. In S. C. Kong, T. L. Wong, M. Yang, C. F. Chow, \& K. H. Tse (Eds.), Emerging practices in scholarship of learning and teaching in a digital era, (pp. 55-74).

Murthy, S., Warriem, J., Sahasrabudhe, S., \& lyer, S. (2018). LCM: A model for planning, designing and conducting learner-centric MOOCS, (p. 73). Chennai: 2018 IEEE Tenth International Conference on Technology for Education (T4E). https://doi.org/10.1109/T4E.2018.00022.

Ogata, H., Majumdar, R., Akçapınar, G., Hasnine, M., \& Flanagan, B. (2018). Beyond learning analytics: Framework for technology-enhanced evidence-based education and learning. Mumbai: 26th international conference on computers in education (ICCE2018).

Ogata, H., Yin, C., Oi, M., Okubo, F., Shimada, A., Kojima, K., \& Yamada, M. (2015). E-book-based learning analytics in university education. In Proceedings of the 23rd international conference on computers in education, (p. 401). China: Asia-Pacific Society for Computers in Education. https://doi.org/10.1007/978-3-319-55345-0-13.

Raba, A. (2017). The influence of think-pair-share (TPS) on improving students' oral communication skills in EFL classrooms. Creative Education, 8, 12-23.

Rachel, B., Di, X., Jihyun, P., Renzhe, Y., Qiujie, L., Bianca, C., ... Padhraic, S. (2020). The benefits and caveats of using clickstream data to understand student self-regulatory behaviors: Opening the black box of learning processes. International Journal of Educational Technology in Higher Education volume, 17(13), 1-24.

Ramma, Y., Bholoa, A., Watts, M., \& Nadal, P. S. (2018). Teaching and learning physics using technology: Making a case for the affective domain. Education Inquiry, 9(2), 210. https://doi.org/10.1080/20004508.2017.1343606.

Rasheed, A., Rasheed, A., \& Kamsin, N. A. (2020). Challenges in the online component of blended learning: A systematic review. Computers in Education, 144, 103701.

Ray, R. K. (2017). Geometry of crystals Retrieved from NPTEL: https://nptel.ac.in/courses/112106227/. 
Sadaghiani, H. R. (2011). Using multimedia learning modules in a hybrid-online course in electricity and magnetism. Physical Review Special Topics - Physics Education Research, 7, 010102. https://doi.org/10.1103/PhysRevSTPER.7.010102.

SantiramKal, I. K. (2004). Introduction to MEMS \& Microsystem Retrieved from NPTEL: https://nptel.ac.in/courses/117105082/.

Stelzer, T., et al. (2010). Impact of multimedia learning modules on an introductory course on electricity and magnetism am. Journal de Physique, 78, 755.

Tanel, Z., \& Erol, M. (2008). Effects of Cooperative Learning on Instructing Magnetism: Analysis of an Experimental Teaching Sequence. Latin-American Journal of Physics Education, 2(2), 124-136.

Teherán, P., Carriazo, J. G., \& León, J. C. (2010). Blended learning applied to an introductory course on conceptual physics. International Journal of Online Engineering, 6, 50. https://doi.org/10.3991/ijoe.v6i3.1303.

Trowler, V., \& Trowler, P. (2010). Student engagement evidence summary. York: The Higher Education Academy.

Y.lmaz, O., \& Malone, K. L. (2020). Preservice teachers perceptions about the use of blended learning in a science education methods course. Smart Learning Environments, 7, 1-21.

Zhang, P., Ding, L., \& Mazur, E. (2017). Peer instruction in introductory physics: A method to bring about positive changes in students' attitudes and beliefs. Physical Review Physics Education Research, 13(1), 010104. https://doi.org/10.1103/ PhysRevPhysEducRes.13.010104.

\section{Publisher's Note}

Springer Nature remains neutral with regard to jurisdictional claims in published maps and institutional affiliations.

Submit your manuscript to a SpringerOpen ${ }^{0}$ journal and benefit from:

- Convenient online submission

- Rigorous peer review

Open access: articles freely available online

High visibility within the field

- Retaining the copyright to your article

Submit your next manuscript at $>$ springeropen.com 